\title{
Mapping research fields using co-nomination: the case of hyper-authorship heavy flavour physics
}

DOI:

10.1007/s11192-020-03538-x

\section{Document Version}

Accepted author manuscript

Link to publication record in Manchester Research Explorer

\section{Citation for published version (APA):}

Karaulova, M., Nedeva, M., \& Thomas, D. (2020). Mapping research fields using co-nomination: the case of hyperauthorship heavy flavour physics. Scientometrics. https://doi.org/10.1007/s11192-020-03538-x

\section{Published in:}

Scientometrics

\section{Citing this paper}

Please note that where the full-text provided on Manchester Research Explorer is the Author Accepted Manuscript or Proof version this may differ from the final Published version. If citing, it is advised that you check and use the publisher's definitive version.

\section{General rights}

Copyright and moral rights for the publications made accessible in the Research Explorer are retained by the authors and/or other copyright owners and it is a condition of accessing publications that users recognise and abide by the legal requirements associated with these rights.

\section{Takedown policy}

If you believe that this document breaches copyright please refer to the University of Manchester's Takedown Procedures [http://man.ac.uk/04Y6Bo] or contact uml.scholarlycommunications@manchester.ac.uk providing relevant details, so we can investigate your claim.

\section{OPEN ACCESS}




\title{
Mapping Research Fields Using Co-Nomination: The Case of Hyper-Authorship Heavy Flavour Physics
}

\author{
Maria Karaulova ${ }^{1}$ \\ ORCID ID 0000-0002-6596-6223 \\ 1. Manchester Institute of Innovation Research, Alliance Manchester Business School, University of Manchester, \\ Booth Street West, Manchester, M15 6PB, UK \\ Maria Nedeva* 1,2 \\ 1. Manchester Institute of Innovation Research, Alliance Manchester Business School, University of Manchester, \\ Booth Street West, Manchester, M15 6PB, UK \\ 2. Department of Business Administration, Research Policy Group, Lund University School of Economics and \\ Management, Lund University, Box 7080, 220 07, Lund, Sweden
}

Duncan A. Thomas 3,4

3 Aarhus University, Department of Political Science, Danish Centre for Studies in Research and Research Policy, Bartholins Allé 8, 8000 Aarhus C, Denmark

4 Nordic Institute for Studies in Innovation, Research and Education (NIFU), PO Box 2815, Tøyen, N-0608 Oslo, Norway

* Corresponding author, maria.nedeva@manchester.ac.uk

* Authors contributed equally and authorship is in alphabetical order.

\begin{abstract}
This paper introduces the use of co-nomination as a method to map research fields by directly accessing their knowledge networks organised around exchange relationships of intellectual influence. Co-nomination is a reputation-based approach combining snowball sampling and social network analysis. It compliments established bibliometric mapping methods by addressing some of their typical shortcomings in specific instances. Here we test co-nomination by mapping one such instance: the idiosyncratic field of CERN-based heavy flavour physics (HFP). HFP is a 'hyper-authorship' field where papers conventionally list thousands of authors alphabetically, masking individual intellectual contributions. We also undertook an illustrative author co-citation analysis (ACA) mapping of 2,310 HFP articles published 2013-18 and identified using a simple keyword query. Both maps were presented to two HFP scientists for commentary upon structure and validity. Our results suggest co-nomination allows us to access individual-level intellectual influence and discern the experimental and theoretical HFP branches. Co-nomination is powerful in uncovering current and emerging research specialisms in HFP that might remain opaque to other methods. ACA, however, better captures HFP's historical and intellectual foundations. We conclude by discussing possible future uses of conomination in science policy and research evaluation arrangements.
\end{abstract}

\section{Article Highlights}


- The paper presents co-nomination approach and explains how it can be used to map fields of science and communities that are hard to access through codified sources

- CERN-based heavy flavour physics, a hyper-authorship field within particle physics, is used as the empirical case study

- The paper maps heavy flavour physics using co-nomination and author co-citation, then deliberates about the merits of each approach

Keywords: science mapping; social network analysis; co-nomination; research field's intellectual influence; heavy flavour physics; hyper-authorship 


\section{Introduction}

This paper describes the use of co-nomination to delineate and map a 'hyper-authorship' research field in physics by accessing directly its knowledge network organised around exchange relationships of intellectual influence; a potentially important methodological innovation. We set out to test this approach by mapping a particularly idiosyncratic global research field conducting expensive, cutting-edge science: CERN-based heavy flavour physics (HFP). This is a highly collaborative, hyper-authorship field publishing large volumes of papers using a publication convention to list hundreds or thousands of authors in strict alphabetical order. This masks individual intellectual contributions (Birnholtz 2008) and strains alternative field-mapping methods.

Much work on studies of research policy and science dynamics ultimately addresses 'research fields' (Noyons, 2004; Porter and Rafols, 2009; Braam and van den Besselaar, 2014; Langfeldt et al. 2020). For instance, a research field is often the unit of analysis chosen to assess how changes in policy, funding or organisational conditions affect research content. And yet we find research fields rarely robustly defined. Instead they are often erroneously conflated with 'disciplines' or their dynamics are overlooked by labelling them retrospectively using static taxonomies or keywords. In this paper we assume research fields must be determined empirically, by mapping them, for their character and type to be usefully understood and interpreted.

Mapping research fields is not a trivial task. Currently, this is done primarily by using bibliometrics based techniques (Lee 2008; Porter and Rafols 2009). We use co-nomination, a reputation-based approach building on snowball sampling (Georghiou 1998; Nedeva et al. 1996) instead to map and analyse the structures of research fields. These structures are understood as sets of relationships based on an exchange (Wellman and Berkowitz 1988). Conomination has certain methodological and resource concerns but, as we demonstrate, it overcomes important general and research field-specific problems associated with the use of bibliometrics.

Scholarly interest in methods to outline and map research fields stems from growing desires to understand and better analyse the many nuances of the multi-faceted and interrelated policy, social and epistemic dynamics of the global science system (Van Raan and Tijssen 1993; Heimeriks et al. 2003; Creswell 2009; Porter and Rafols 2009; Boyack and Klavans 2014). Existing approaches have largely remained rooted in bibliometrics-based techniques, like bibliographic coupling and co-citation analysis. These can be useful to delineate and analyse research fields, and their evolution over time, but have recognised limitations. Bibliometrics uses publication proxy data to evaluate research. Methodologically, the same technique may yield different results depending on the databases used for analysis (Meho and Yang 2007). Citation data most frequently used in analyses is also only a proxy of research quality, conveying just one type of intellectual link between authors, and only acknowledging contributions of knowledge community members writing the research.

The limitations of citation-based methods are especially evident in research fields with unconventional publishing and complex authorship practices. A prime example is fields with hyper-authorship, where typically papers have above 1,000 authors (Cronin 2001) although articles with more than 100 authors and/or 30 countries can also be defined this way (Adams et al. 2019). There has been a rise in hyper-authorship, still largely, in clinical medicine and particle physics (Adams et al. 2019). After scoping apparent intellectual and organisational features of various areas of particle physics, and finding conventional tools lacking to 
understand their intellectual influence structures, we settled on HFP as a suitable candidate to explore further.

In HFP, similar to other fields in particle physics, a prerequisite for using colossally expensive, large-scale CERN infrastructure funded by collaborative international agreements is that scientists must publish in strict, convention-based, alphabetically listed author collectives. Publications can have hundreds or thousands of contributing authors. Articles may collect high numbers of citations, but intellectual credit is hard to attribute to individual authors, author groupings or author host organisations, and remains opaque to most citation-based mapping techniques. Previously, authors attempted to use surveys to alleviate the opacity of particle physics to bibliometric tools (Bellotti 2011; Canals et al. 2017). However, these studies were limited by their data sources: one experiment or one country. Their results are therefore incomplete and cannot be used to map and analyse the intellectual structure of an overall research field. We chose to explore a co-nomination approach instead to address these limitations.

The co-nomination method combines snowball sampling and social network analysis that, we demonstrate, can reveal intellectual influence and collaboration structures even in hyperauthorship fields. It can also substitute, or complement, citation-based metrics, ascertain sources and distribution of intellectual influence, and capture cognitive and social dynamics. Co-nomination has already been used to map expert communities and scientific fields before modern advances in computing made citation data readily available for the now more popular bibliometrics based approaches (Shrum \& Mullins 2013; Crane 1971; Libbey \& Zaltman 1967). It has also been more recently used within mixed methods to map 'cognitive colleges' around research areas in vaguely defined interdisciplinary research fields (Degn et al. 2019), and in network analysis for systemic foresight approaches (Saritas \& Nugroho 2012).

This paper contributes to the literature on outlining and mapping research fields in two ways. First, we use co-nomination to map a research field by accessing its knowledge network directly and in real time. This approach, we believe, avoids inferring relationships from analysis of science artefacts (publications) and instead captures them by studying specified types of exchange between members of the network. It also opens assessment of intellectual influence where field authorship conventions and citing behaviour occlude cognitive links between researchers. It further allows us to consider issues like, for instance, whether formal leadership in such large collaborations correlates with intellectual influence. Second, we assess the strengths and weaknesses of the co-nomination approach by presenting our resulting research field maps to two HFP scientists, for commentaries on structure and validity. Ordinarily the use of a novel method is also assessed by comparing the results to those achieved by using a well-established approach which in this case is problematic, as we will discuss.

The next section will address some definitions and our assumptions about research fields, knowledge communities and networks, and provide a brief description of the HFP field. We then present our methodology and approach to the HFP co-nomination mapping and discuss the preparation of our illustrative author co-citation analysis (ACA) map of HFP. Finally, we discuss the method verification based on the commentaries from HFP scientists, before concluding by exploring some implications of our method development for studies of science policy and research evaluation arrangements.

\section{Context and analytical assumptions}

\subsection{Working definition of a 'research field'}


The notion of 'research field' is a way to conceptualise and access empirically certain dynamics of the science system. The term 'research field' remains widely used but often not well defined. Here we anchor our understanding in the social and epistemic organisation of science (Whitley 2000) and Nedeva's conceptualisation of the science system as a matrix relationship between policy and funding 'research spaces' and 'research fields' (Nedeva 2013; Nedeva 2010). We also distinguish 'research fields' from 'disciplines'.

Whitley (2000) defines research fields as: 'reputational units of research work organisation which reward innovative contributions to collective intellectual goals; control material rewards through public reputations; combine collegiality with competition; and direct research to achieving intellectual influence' (p. 34). This foregrounds three characteristics of research fields: they are reputational; they are a form of organisation; and they are organised around collective intellectual goals that determine material and intellectual rewards. Nedeva (2013) extends this work by seeing research fields as 'outlined by three inter-connected elements, namely relatively converging knowledge communities, intellectually, methodologically coherent bodies of knowledge and research organisations' (p. 221). This foregrounds knowledge communities, which are further organised as knowledge networks crystallising or integrating around, amongst other things, coherent bodies of knowledge (Luukkonen \& Nedeva 2010).

\section{'Knowledge communities' accessed through 'knowledge networks'}

Work on research fields can be traced back to the concept of 'scientific community' in the sociology of science from the 1960s and '70s. Merton's normative structure of science, for instance, outlined the broad rules to ensure healthy scientific communities emerge, thrive then recede (Merton 1968). Norman Storer used the notion of 'scientific community' to analyse the social organisation of science as an exchange system that develops specific rules of exchange and legitimacy (Storer 1966). Later, Diana Crane (1971) carried out one of the most influential studies of scientific communities and examined whether and how scientific communities affected knowledge growth. This study defined 'scientific communities' as communication networks among scientists, structured by exchanges of information. Different research traditions have subsequently made divergent assumptions regarding the rules and underpinning principles of these exchanges (see Knorr-Cetina, 1982).

This brings us to 'epistemic' or 'knowledge' communities. There is no universally accepted definition here but there is a tentative agreement that epistemic/knowledge communities empirically are: a) defined by relationships between their members; b) characterised by shared systems of beliefs, rules and in some cases epistemic standards and practices; and c) organised around particular mechanisms of authority. Hence, Haas (1992) defines an epistemic community as 'a network of professionals with recognised expertise and competence in a particular domain and an authoritative claim to policy-relevant knowledge within that domain or issue area' (p. 3). Fagerberg and Verspagen (2009) later used this notion of epistemic communities to study the emergence and development of the field of innovation studies. They highlighted organisational elements needed to legitimise a research field, like a 'separate communication system' including 'conferences and journals, common standards ... and a merit-based reward system’ (p. 219).

To underpin our understanding of a 'research field' we adopt a composite notion of 'knowledge community'. We see it as a form of social organisation in science characterised by shared rules and standards. Knowledge communities organise around coherent bodies of knowledge. They can include members with different backgrounds so long as they take part in the knowledge 
production processes (in some capacity) and subscribe to established (albeit continuously negotiated) community rules. Knowledge communities are structured by a variety of exchanges, including, but not limited to knowledge, information, resources, and reputation. Alongside bodies of knowledge and research organisations, we believe that knowledge communities are central to any definition and delineation of 'research fields' (Nedeva 2013).

We further assume that research fields are not necessarily institutionalised (Braun 2012). This distinguishes them from the academic disciplines. This lower degree of institutionalisation of research fields and weak association with research organisations leads us to knowledge communities themselves - and the bodies of knowledge around which they integrate - as the two preferred empirical entry points to map, analyse and study 'research fields'.

Bibliometrics based approaches, we assert, use bodies of knowledge as their empirical entry point, using citation data as a proxy to infer intellectual structure. This constitutes a metricsbased mapping. Our methodology instead proposes a relational mapping approach. Conomination we believe can access directly research field structure by mapping the knowledge network of exchange relationships of the field's knowledge community. Our method choice is therefore to map a network of relationships, by identifying forms of exchange between members of a knowledge community. ${ }^{1}$

Our study mapped three research field structures, i.e. intellectual influence, collaborative patterns and relationships in relation to research equipment. These were selected to inform our broader intellectual aims. However in this paper, we use only the first, i.e. the HFP structure of intellectual influence to illustrate use of the method.

\subsection{CERN-based high energy physics (HEP) and heavy flavour physics (HFP) field}

Heavy flavour physics (HFP) is a research field within the broader area of high energy physics (HEP). HEP has a wide set of aims to find proof for science beyond the Standard Model (SM) of physics, to explain fundamental questions about the Universe (Heuer 2012) and to refine characterisations of the SM fundamental 'particle zoo' (Campanelli 2015). HEP is very costly. Most of its experiments rely on unique trans-national facilities, most notably the long-standing and continually upgraded European Organization for Nuclear Research's Large Hadron Collider (CERN LHC).

HEP has both experimental and theoretical branches. Experimental HEP uses unique purposebuilt beam devices to create and study the results of high energy particle collisions. Theoretical HEP addresses numerical simulations and applied mathematics to predict and interpret experimental results from these beam collisions and subsequent particle decays. These two core branches of HEP have distinct knowledge production, publication practices and reputation control mechanisms (Lehmann et al. 2003). Theory groups are often small, relatively shortlived and globally dispersed whereas experimental groups crystallise around CERN. CERNbased collaborations involve up to several thousand researchers employed by universities and research organisations but often spending periods onsite at CERN. These research collectives are relatively stable over time and have a formally organised division of labour around often

\footnotetext{
${ }^{1}$ Here we necessarily disregard other layers to the notion of knowledge community we have been discussing, such as values, normative and social control aspects. These would require other, more qualitative and extensive methods to address.
} 
complex and interdependent technical and research tasks, and forward work planning and scheduling.

These collaborations have strong scientific and organisational planning and coordination and funding commitments that can span decades. ${ }^{2}$ Authority is formalised in highly codified reputational control mechanisms, with flowcharts to specify when and who can publish significant scientific results. This formal, transparent and rigorous internal scrutiny seems to generate high levels of institutional trust in non-peer reviewed pre-prints, which are the main source of scholarly information exchange in HEP, in advance of subsequent international journal publication. ${ }^{3}$

HEP decision-making mechanisms are communitarian (Knorr-Cetina 1999). This generates strong, persistent boundaries that are likely to be reflected in the knowledge networks we will map say, between members and non-members of experiments, between experimentalists and theoreticians, between residential and visiting physicists. ${ }^{4}$ These will feature various forms of interesting intellectual exchange network dynamics within HEP. Authorship rules somewhat reflect the intellectual authority over the equipment-based collective of participating physicists and institutions, the 'scientists' (physicists), technicians/engineers, 'authors' and non-authors.

Scientific outputs are published under a group name representing all scientists in that CERNbased collaboration, listed in a strict alphabetical order. This perhaps deliberately subsumes and renders impossible individual attribution of intellectual contribution, credit and accumulation of reputation beneath the collective nature of the science (Birnholtz 2008; Cronin 2001). Being included in CERN-based HEP authorship on articles means publishing multiple articles every week. Within such large author collectives reputational attainment occurs through informal channels that are opaque to citation-based query, such as 'getting noticed' (Birnholtz 2006). Researchers' competencies are recognised by awarding them opportunities to deliver seminars or present at conferences. Academic reference letters are also particularly important. Specialised, exclusive field-only conferences bring theorists and experimentalists together, whilst smaller scale workshops facilitate discussion of specific scientific developments and topics.

Within HEP, heavy flavour physics (HFP) itself is distinctive in its intellectual specialisation rather than in its social or resource characteristics. HFP scientists pursue similar overarching scientific aims as broader HEP but focus specially on high precision measurements of decays of hadrons involving charm and beauty (a.k.a. bottom) quarks (Gershon \& Needham 2015). These measurements are complementary to direct searches for new physics on the so-called energy frontier (Lambert 2011). They determine which particles can be generated, studied

\footnotetext{
${ }^{2}$ Membership in the LHC experiments is institutional, not country based. Member organisations are obligated to make annual contributions towards operating costs and towards the costs of equipment upgrading. The required amount of the contribution changes depending on the number of scientific authors from that organisation in the experiment.

${ }^{3}$ This pre-print culture seemingly emerged to bypass journal processing times. It is now so widespread in HEP that Gentil-Beccot et al. (2010) state journals have 'lost their role as a means of scientific discourse' in HEP and instead constitute merely a 'repository' and 'independent accreditation' for research (p. 354).

${ }^{4}$ Physics analyses at CERN experiments are interpreted and reviewed within the collaboration before they are released publicly. Typically, small teams of scientists responsible for analysis of experimental data will draft and review an initial version of a publication before it is then also peer-reviewed by other physicists in the collaboration.
} 
and/or discovered. The specific LHCb (beauty) detector equipment at CERN is the primary experimental site for HFP, whilst HFP theory groups are dispersed globally.

HFP's 'hyper-authorship' (Birnholtz 2008) co-authoring scale and alphabetised publication conventions make it opaque to map using most bibliometric techniques. These conventions blur individual intellectual contributions and emphasise collectivity. This and the formalised paper writing guidelines change the meaning of citing behaviour beyond intellectual influence and reputation. We explore this in our contrasting of our co-nomination approach with an ACAbased map in our verification interviews with HFP scientists.

\section{Methods and mapping approaches}

We now present our co-nomination mapping approach along with, briefly, our ACA method to generate an additional map of HFP for illustrative purposes only, to contrast the two for our HFP scientist interviewees.

\subsection{Co-nomination approach to map HFP (relational approach)}

We applied co-nomination using an online survey-based approach. First, we directly asked survey participants to self-identify as HFP field members and, second, to nominate intellectually influential figures in HFP. We initiated the survey from a starter set of group members ('nominators'). These members were asked to identify 'nominees' then the chainreferral sampling process was repeated until few new names appear (Borgatti et al. 2018). This process is summarised in Figure 1. The frequency with which persons are nominated is then linked to the strength of their intellectual influence in the HFP field. ${ }^{5}$

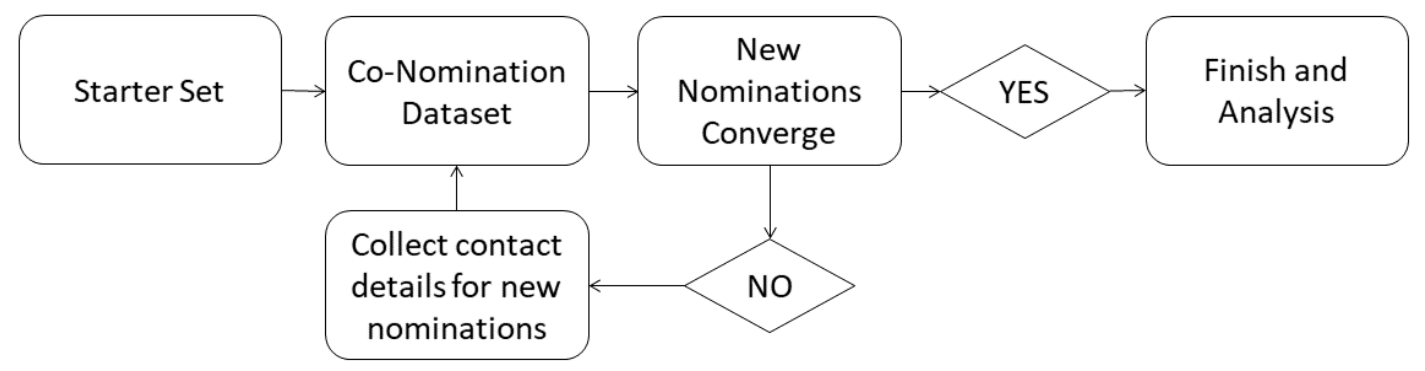

Figure 1. Flowchart of the co-nomination process. Source: Authors.

\footnotetext{
${ }^{5}$ We recognise that networks sampled in this way are never complete, but they do provide insights about overall network structure and these networks can be quantitatively analysed. Co-nomination networks have robust degree centrality (Costenbader \& Valente 2003) and represent core structural relations between members after a certain number of nomination rounds (Wejnert 2010). Co-nomination is also useful to study emic social groups with fuzzy boundaries, as self-identified by their members. Co-nomination has also been employed successfully to identify participants of the UK Technology Foresight Programme to identify expert networks around a variety of topics (Nedeva et al. 1996).
} 
This method assumes intellectual proximity and semantic link between nominees (see Figure 2). This enables identification of clusters of intellectual influence within the research field, and links to proximate research fields. ${ }^{6}$

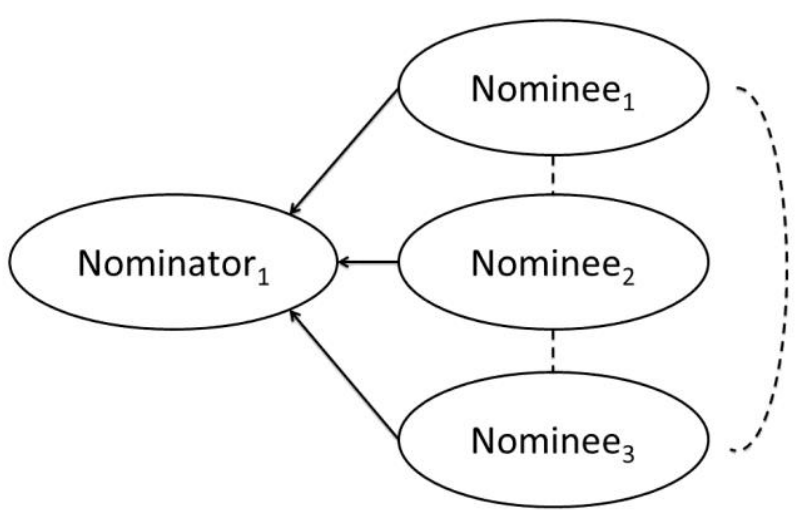

Figure 2. Co-nomination assumptions. The solid line represents assumed intellectual influence; dashed represents assumed intellectual proximity. Source: Authors.

The co-nomination network can be understood both qualitatively and by applying network statistics. Structure and degree centrality measures of the network can be analysed, and 'elite' nodes and major intellectual groupings can be identified. A key advantage of using conomination to map HFP is that it bypasses the problems associated with hyper-authorship collectives we have mentioned.

To familiarise ourselves with HFP we first undertook an ex ante characterisation, through desk research and a scoping interview with one HFP scientist. We learned HFP researchers do not have dedicated journals or conferences, but convene regularly at three workshops that are key 'meeting places' for HFP field members. We sampled the co-nomination starter set (the seed nominators) from lists of researcher participants at recent sessions of these workshops (see Table 1). ${ }^{7}$ E-mail addresses were collected from public sources. We compiled the names and contact details of 516 unique scientists in this starter set, and believe it captured the majority of these key workshop participants.

\footnotetext{
${ }^{6}$ The resulting network of linkages is not dissimilar to an ACA network, in which author pairs are counted as semantically linked if cited together in an article. No systematic examination has yet been performed about the similarity of outcomes obtained through these different methods. An analysis by Giusti and Georghiou (1988) suggests co-nomination mapping yields similar results to those derived from citation-based scores, for fields where the two can be directly compared.

${ }^{7}$ The number of workshop participants ranged from 80 to over 200. These workshops were held in the Austria, France, India, Italy, the UK and the USA.
} 


\begin{tabular}{cccc}
\hline Title & Topic & Dates & Names Sampled \\
CHARM & Charm quark physics & $2013,2015,2016$ & 241 \\
BEAUTY & Beauty quark physics & 2014,2016 & 173 \\
CKM & Weak interactions in CKM & 2014, 2016 & 325 \\
Workshop & unitarity triangles & & \\
\hline
\end{tabular}

Table 1. Starter dataset collection sources. Sources: Beauty Workshop 2014; Beauty Workshop 2016; CHARM Workshop 2013; CHARM Workshop 2015; CHARM Workshop 2016; CKM Workshop 2014; CKM Workshop 2016.

We then developed a deliberately parsimonious and precise online questionnaire with very specific questions to capture the kinds of exchange of intellectual influence of interest to us. This was a key methodological step. We wished to map the HFP field in terms of intellectual, social and resource links (after Whitley 2000). We developed a single question for each of these three types of exchange (see our nomination questions, Table 2) along with five 'passport' questions (to confirm research field affiliation, career stage, gender, academic age, and place of $\mathrm{PhD}$ completion, where applicable). Completion of all question fields was optional. Respondents could nominate up to five people for each question. We also asked them to provide a contact email address and organisational career stage for their nominees. The emailed survey invitation asked nominees to participate only if they self-identified as being involved in HFP research.

\section{Type of Influence}

Direct Intellectual

Influence

Field Social

Structure

Technical

Expertise

\section{Co-Nomination Question}

Can you please name up to five people that influence you intellectually at the moment?

Can you please name up to five people with whom you most recently discussed a research problem you want to study?

Can you please name up to five people with whom you recently discussed the technical design of your research project? (think about one specific project)

Table 2. Co-nomination questionnaire survey questions.

Co-nomination data was collected from October 2017 to March 2018 (see Table 3). After receiving responses from the starter set, we selected names new to the dataset then emailed participation invitations to those scientists (emails were identified for $89.5 \%$ of the nominated scientists). This was repeated until the share of newly nominated persons fell to $30 \%$. Each round of survey data collection consisted of an initial invitation to participate followed by two 
reminders, with a week between each step in this process. ${ }^{8}$ We ran five nomination rounds, garnering progressively fewer new nominations after round three.

\begin{tabular}{cccccc}
\hline & Round 1 & Round 2 & Round 3 & Round 4 & Round 5 \\
Responses & 86 & 93 & 71 & 32 & 9 \\
Response Rate & $17.7 \%$ & $28.3 \%$ & $22.3 \%$ & $12.5 \%$ & $10 \%$ \\
\hline
\end{tabular}

Table 3. Co-nomination data collection results.

Overall 1,479 persons were invited to complete the survey; 291 answered (19.7\% response rate). For our open-ended question, respondents self-identified as particle physicists: $88 \%$ of respondents said their research was a type of 'physics'; 55\% mentioned 'particle' or 'energy' physics. Equal shares of respondents additionally described their field as 'theory/theoretical' and 'experiment/experimental' ( $13.7 \%$ and $13.4 \%$, respectively). $89 \%$ of respondents were male. Most responses (40\%) came from senior researchers; early/mid-career scientists were about half the responses. There was a median respondent academic age of 14.5 years, and median HFP field experience time of 15 years. This longer experience time versus academic age suggests our respondents were inducted into HFP publishing before completing their doctoral degrees and probably did not later change specialisation. Respondents identified as coming from 29 countries, with the largest shares from the USA (17.2\%), Italy (13.7\%), Switzerland (8.9\%), Germany (8.9\%) and the UK (8.6\%). ${ }^{9}$

A total of 2,823 nominations was received across the three questions. Here we focus on nominations received for Question 1, addressing direct intellectual influence. 1,063 nominations came from Question 1, with 671 unique names and 1,691 co-nomination pairs. Our co-nomination matrix was generated for the 538 nodes and 1,482 links constituting the two main components of the network (out of the 35 total). For structure analysis, we calculated the Louvain modularity statistic and performed Johnson's hierarchical clustering (Johnson 1967).

To help interpret the co-nomination network results, we interviewed two HFP scientists. These interviewees were identified through convenience sampling with purposive elements, considering interviewee specialisation, career stage and experience in scientific outreach. For the first interview we returned to the mid-career HFP experimentalist with whom we had conducted our initial HFP field characterisation scoping interview (labelled I1 below). This interviewee nominated a knowledgeable senior HFP theorist from a different organisation

\footnotetext{
${ }^{8}$ This implies around 20 weeks in total to deploy this approach (i.e. a minimum 4 weeks per round, which could be extended to allow for additional response time following the final reminder). Overall, we took six months to follow this approach because a holiday period also fell in the middle of our study.

${ }^{9}$ Ten countries had ten or more responses, including China, India, France, the Netherlands, and Japan. The major country-level representation broadly corresponds with both volumes of HFP publications and accumulated citation scores, except for India. We estimate over-representation of survey respondents from India was a characteristic of our starter sample. The 2016 CKM workshop was held in Mumbai and was attended by local scientists. However we did not find an over-representation of Indian scientists amongst our nominees.
} 
(labelled I2). For these two interviews we wanted to be able to contrast and discuss the merits of our co-nomination, relational mapping of HFP with a more conventional, metrics-based approach. To do this we undertook a simplified mapping, strictly for illustrative purposes, using ACA.

\subsection{An illustrative mapping of HFP using ACA (metrics-based approach)}

Metrics-based approaches to map research fields access them via bodies of knowledge, indirectly, using metrics to assess scale, scope, research quality, and to infer relationships between members of the knowledge network. For illustrative purposes limited to our interviews with HFP scientists we used ACA to generate a metrics-based map of HFP - the most proximate approach we believe, in terms of aims, to co-nomination.

ACA can map individual level intellectual influence in research fields by identifying 'influential authors and display[ing] their interrelationships' (White \& McCain 1998, p. 327).

The basic co-citation technique counts the number of times two authors are cited together, regardless of number of times their particular articles are co-cited (White \& Griffith 1981). ACA links intellectual and social structures of research fields, especially how these evolve, and the role of research traditions and seminal authors (Nerur et al. 2008). ACA is strong in showing the intellectual foundations of a research field and how they relate to each other structurally (Zhao \& Strotmann 2008).

Using ACA even for our illustrative purposes however encounters methodological and computational limitations. These not only affect the quality of the resulting network, but also help us understand what ACA mapping does and does not allow, and where its inherent weaknesses indicate co-nomination can be complementary.

In generating the ACA matrix, we sought to obtain a contemporary view of the HFP field. We collected 2,310 HFP publications from general particle physics journals indexed in the Web of Science database from 2013-18 (inclusive). We chose only to collect article document types, as our scoping work had shown these are the primary means of scholarly communication in HFP. Hence, collecting only articles would be sufficient to illustrate strengths and limitations of ACA here. We used a Boolean search query (see Appendix 1). Next the names of 3,479 first authors were extracted from 47,807 references. Due to the long author lists per article, only the first author co-citation matrix was generated.

The most cited authors are presented in Table 4. The ACA network for authors with 10 or more citations is in Figure 3. The ACA network map of the field has 1,974 author nodes, and 386,136 links representing instances of co-citation.

\begin{tabular}{cccc}
\hline \multicolumn{1}{c}{ HFP Author } & Affiliation & Type & Times Cited \\
\hline Particle Data Group & $\begin{array}{c}\text { Lawrence Berkeley Natl } \\
\text { Lab }\end{array}$ & Group & 1253 \\
\hline LHCb collaboration & CERN & Group & 858 \\
\hline CMS Collaboration & CERN & Group & 856 \\
\hline ATLAS collaboration & CERN & Group & 792 \\
\hline
\end{tabular}




\begin{tabular}{cccc}
\hline BaBar Collaboration & SLAC & Group & 616 \\
\hline Sjostrand Torbjorn & Lund University & $\begin{array}{c}\text { Individual - } \\
\text { Theory/method }\end{array}$ & 472 \\
\hline CDF Collaboration & Fermilab & Group & 422 \\
\hline Cacciari Matteo & Sorbonne University & $\begin{array}{c}\text { Individual - } \\
\text { Theory/method }\end{array}$ & 381 \\
\hline D0 Experiment & Fermilab & Group & 332
\end{tabular}

Table 4. Most cited authors in HFP. Sources: Web of Science, calculations by the authors. 


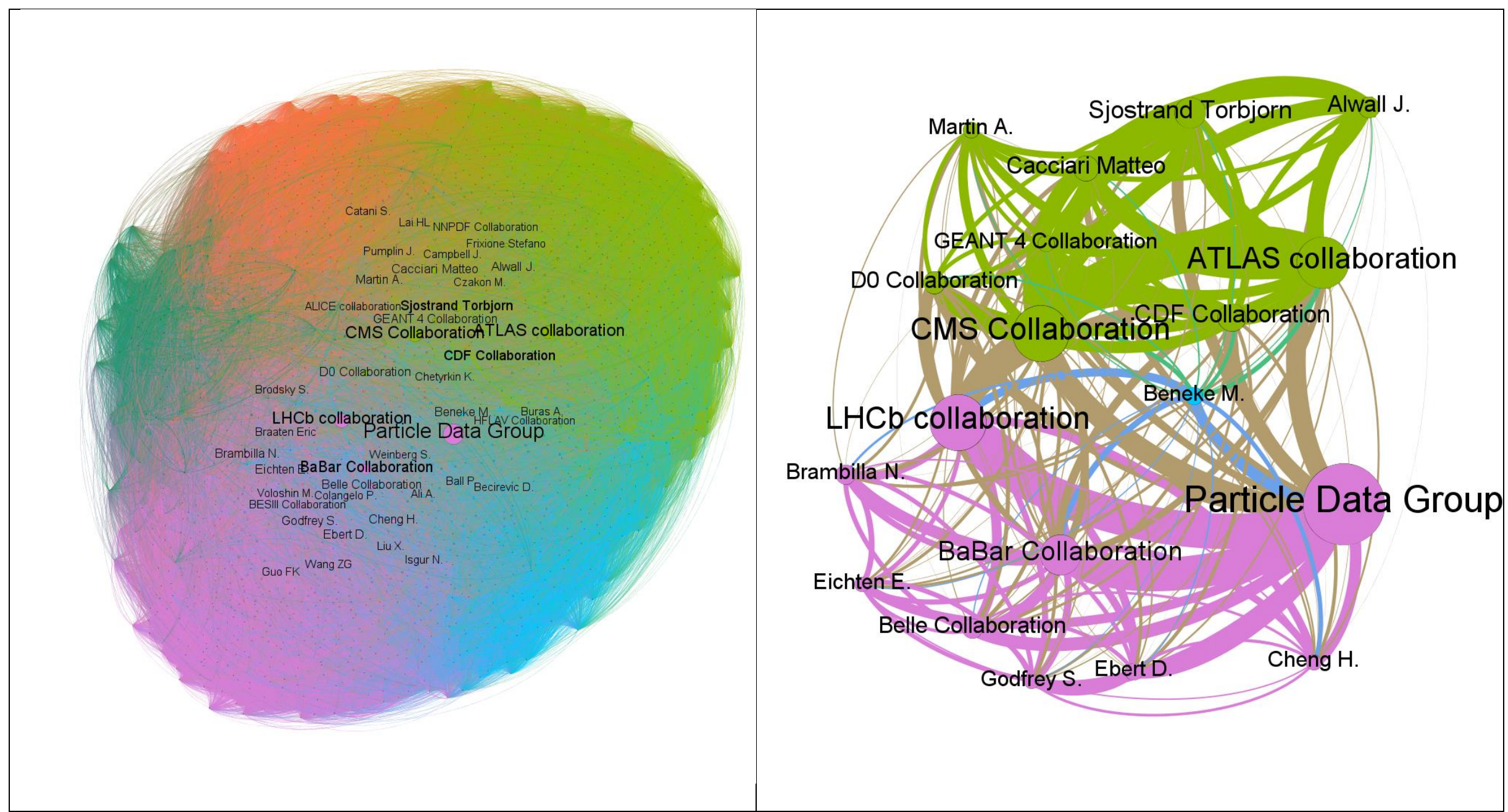

Figure 3. Author co-citation networks of HFP. Left: Network with more than 10 citations. Right: Simplified networks showing authors cited over 210 times. Size of the nodes corresponds to node degree; thickness of edges corresponds to edge weight; colouring is according to a community structure algorithm outlined in Blondel et al. 2008. Networks were calculated and visualised in Gephi 0.9.2. Sources: Web of Science; total N =3,482; Left: $\mathrm{n}=1,974$; Right: $\mathrm{n}=19$; calculations by the authors. 
In Figure 3, nodes with the highest degree, or number of links from and to them, are positioned in the centre of the network. Authors co-cited more often are placed closer to each other. Nodes representing authors with higher citation numbers are scaled up, and link thickness reflects number of times authors were co-cited together in paper references. For clarity, we also visualised the co-citation network of 24 authors whose works were co-cited more than 150 times.

ACA highlights certain intellectual and social features of HFP. As expected, most of the major cited authors in the network are group authors. For example, the Particle Data group (PDG), an international collaboration coordinated by the US Lawrence Berkeley National Laboratory, is the highest cited, followed by three CERN experiments: LHCb, CMS and ATLAS. The PDG group produces a biannual publication, A Review of Particle Physics, compiling and evaluating experimental measurements and searchers for new particles. This explains its high rates of citation. The highest placed individual author is best known for a software programme allowing simulation of complete physics system events. Additionally apparent intellectual influence in HFP here originates in the traditional scientifically dominant countries and research organisations from Europe and USA.

The community structure of the network, calculated using Louvain modularity, highlights areas from broader HEP that provide intellectual foundations for HFP (see Table 5). We can also distinguish between HFP theoretical foundations and experimental, methodological and computational knowledge bases of HFP (i.e. communities 1 and 2). Both are dominated by large experimental collaborations and by individual authors who developed computational tools for both theoretical and experimental HEP. These intellectually connect to the theory of flavour physics (community 3), relevant research in mathematical physics and perturbative quantum field theory (community 4) and other relevant research areas in particle physics, from heavy ion physics to supersymmetry (community 5 ).

\begin{tabular}{cccc}
\hline Cluster No & Nodes No & Label & Colour in Fig 3 \\
1 & 587 & Field Foundations & Purple \\
2 & 555 & Experiments and tools & Light Green \\
3 & 361 & Flavour Physics Theory & Blue \\
4 & 313 & Mathematical and pQFT physics & Orange \\
5 & 158 & Other relevant physics & Dark Green \\
\hline
\end{tabular}

Table 5. HFP ACA communities. Sources: Web of Science, calculations by the authors.

The metrics-based ACA mapping seems largely in line with our expectations. We see the broad intellectual history or foundations of HFP mapped, and overarching areas of broader HEP that intellectually influence HFP. ACA mapping distinguishes theoretical from experimental HFP but does not provide much insight into intellectual influence of researchers advancing the field. Instead, we see only relative positions of various large collaborations, with even individuals who made significant theoretical contributions not visible. Due to the sheer volume of research produced in HFP and its authorship conventions, highly visible individual researchers are ones cited in frequently published experimental research papers, crediting the computational tools 
they developed. ACA, as might be expected, also does not show current or forward-looking HFP research trends. ${ }^{10}$

\section{Results of the co-nomination approach}

We consulted two interviewees to interpret the co-nomination network, and to comment on its structures and validity, based on their informed opinion as active HFP field members. We also contrasted the co-nomination approach HFP field map (Figure 4) with the above ACA network map (Figure 3). Interviewees were free to explore both networks interactively using a computer. Specifically, we asked the interviewees to select which clustering partitions made the most sense to them. In the following section, only the clustering solution they considered the best is discussed (i.e. Johnson's hierarchical clustering).

\subsection{Structures of intellectual influence in the co-nomination HFP map}

Most nominated scientists, and all nominated scientists at the core of the network in Figure 4, self-identify as undertaking research in heavy flavour physics. HEP researchers whose work has influenced the HFP field are visible as the peripheral clusters. None of these individuals, however, approach the core of the field, which is an indicator of a strong field identity. The main component of the network includes over $75 \%$ of all nodes, meaning most scientists in the network have a co-nomination path linking them to other scientists (the average length of this path is 5 and the network diameter is 13$)$. The graph has very low density (0.01) and very high clustering coefficient (0.8). This points to clear separation of the network into distinctive communities. It also indicates a group of nodes central to the network may be crucial for information exchange flows.

${ }^{10}$ Using ACA to map the intellectual influence structure of HFP comes up against the collective authorship conventions and opaque individual contributions of experimental HFP. Research attempting this task so far had to gain access to exclusive data not publicly available and had to limit its scope either to one country (Bellotti 2011) or one particle physics experiment (Canals et al. 2017). Computationally it is also challenging if not impossible to include all paper authors in the ACA network map. We had to limit our analysis to first authors. This constitutes an additional methodological difficulty to map a hyper-authorship research field using this kind of citation-based technique. 


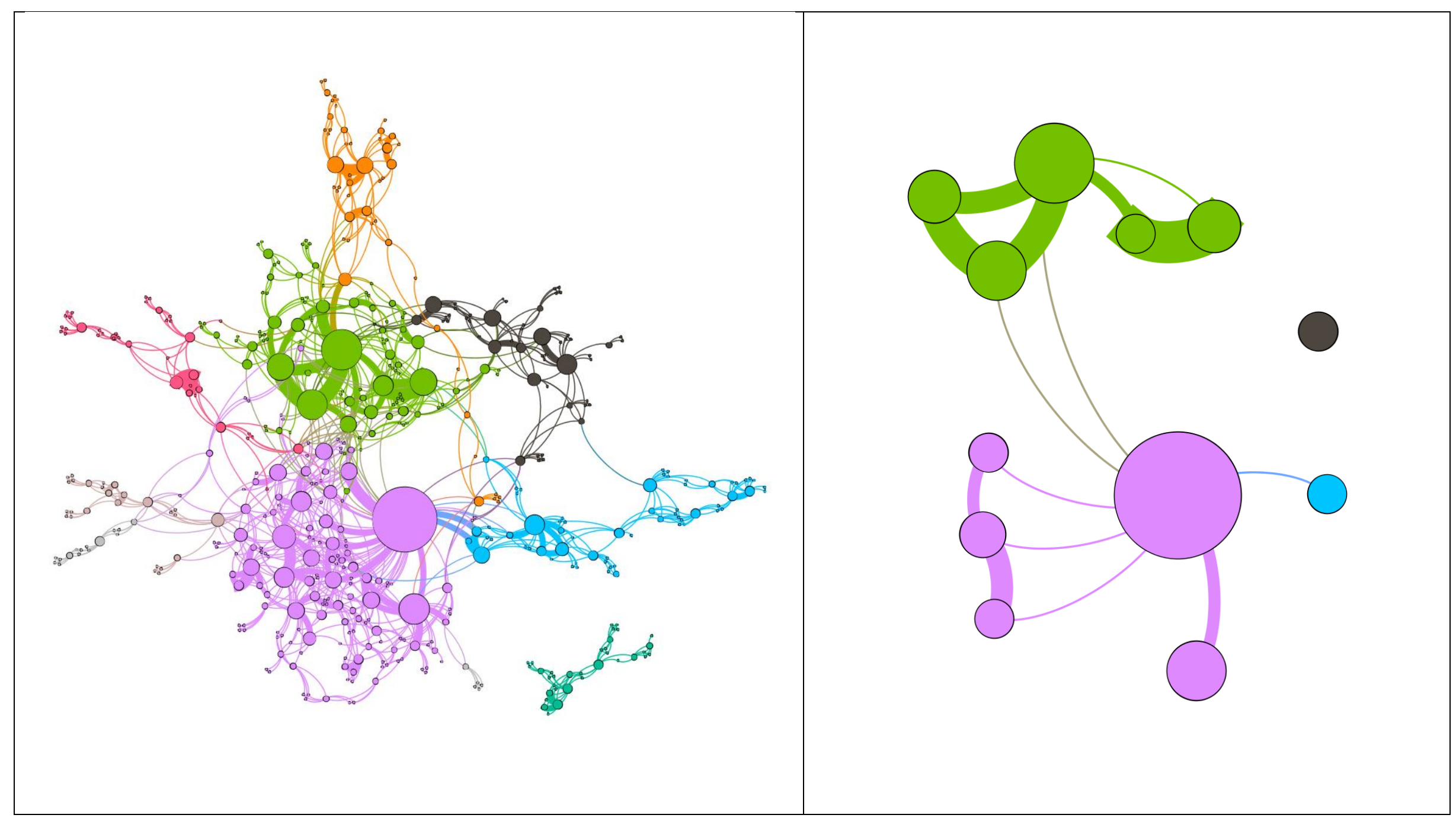

Figure 4. Co-nomination network of the HFP research field. Two major components are shown left; simplified network showing scientists nominated six times or more shown right. Node sizes correspond to node degree; edge thicknesses correspond to edge weight. The chosen layout ForceAtlas 2 places similar nodes close to each other. Colouring is according to hierarchical clustering results (Johnson 1967). Calculated in UCInet 6 and visualised in Gephi 0.9.2. Source: Authors; Left: $\mathrm{n}=538$; Right: $\mathrm{n}=12$. 
Partitions of the network represent HFP theory, experiment and equipment divides. Similar to the ACA results, the biggest partition is between theoretical HFP researchers (positioned mostly in the bottom half of the network) and experimental HFP researchers (top half). We coloured the hierarchical clusters in the co-nomination network, reflecting this best interpretation of community structure of the network that our two interviewees found most meaningful and easy to interpret (see Table 6).

\begin{tabular}{cccc} 
Cluster & Nodes Share & Label & Colour in Fig 4 \\
1 & $28.8 \%$ & Heavy Flavour Theory & Purple \\
2 & $18.6 \%$ & LHCb Collaboration & Light Green \\
3 & $11.7 \%$ & Kaon Physics & Blue \\
4 & $9.7 \%$ & Belle Experiment & Black \\
5 & $9.1 \%$ & ALICE Experiment & Orange \\
6 & $7.3 \%$ & Neutrino Theory & Pink \\
7 & $6.3 \%$ & Lattice Gauge Theory & Turquoise \\
8 & $4.8 \%$ & Higgs Physics Theory & Pale Pink \\
$9-12$ & $3.7 \%$ & Minor clusters & Grey \\
\hline
\end{tabular}

Table 6. HFP Co-nomination network clusters. Source: Authors.

For experimental HFP, the importance of formal collaborations is showcased, because they define the clusters in the network. Compared with the ACA-based map, insight into the role of these collaborations is more nuanced. Particularly, the CERN LHCb experiment (cluster 2) currently highly important for HFP itself - is now central to the network. It is also the main route through which the experimental top half connects to the theoretical bottom half. Linked to $\mathrm{LHCb}$ are groups of scientists working in other experiments at CERN, and in experiments beyond, producing HFP-relevant results. ${ }^{11}$ The LHCb cluster also connects with the only mixed theory and experimental cluster of physicists working on Kaon physics and strange quark physics (cluster 3).

In the bottom half of the network, the Heavy Flavour Theory cluster (cluster 1) is the largest in the network and remains undifferentiated. Our interviewees noted that although there are many important figures among theoretical HFP researchers, there is no real division in terms of specialisations. This indicates perhaps there is no need for further differentiation between the centres of intellectual influence in theoretical HFP. Theoretical HFP is informed by other areas of HEP like Higgs (cluster 8) and Kaon physics (cluster 3). Communication between theory and experiment in HFP is seemingly achieved through a small number of 'broker' scientists.

\footnotetext{
${ }^{11}$ E.g. the CERN-ALICE experiment measuring heavy quarks (cluster 5) and the now-concluded Belle experiment at the KEK facility in Japan, whose purpose was to produce and measure b-mesons (cluster 4).
} 
Most importantly, the co-nomination map allows us to analyse sources and relations of individual-level intellectual influence in HFP. There are two such major sources on the map: an individual HFP theorist (cluster 1), and a group of interlinked experimentalists from the LHCb collaboration (cluster 2). The most nominated scientist in the network (19 nominations) is a European theoretical HFP professor. Although this person is considered 'very influential intellectually', their work is not 'very highly cited' such as to be highlighted by metrics-based approaches. This scientist had 'proposed several theories' driving speculative, cutting-edge investigations but not yet supported by positive, publishable experimental results (I1). ${ }^{12}$

The second major source of intellectual influence is distributed across five interconnected nodes in cluster 2 (12, nine, nine, eight and six nominations). These are experimental physicists occupying governance positions in the LHCb experiment. Our interviewees associated being in such positions with ability to exert influence over future directions for LHCb. At the same time, they did not see reaching such a position as a simple progression through the ranks and gaining experience, but rather a result of talent and creativity.

There are more nodes with higher individual intellectual influence in the theoretical, bottom, half of the network than in the experimental half. Influential HFP theorists are all active researchers who have made significant contributions to the field, including study of strong and electroweak particle interactions, and theoretical analyses of $\mathrm{CP}$ violation (matter-antimatter asymmetry). This is perhaps associated with a seemingly greater need for HFP theorists, as opposed to HFP experimentalists, to build individual reputational portfolios. Such differences in individual-level analysis of the network point to potential differences in intellectual influence attainment pathways in experimental versus theoretical parts of HFP. This observation - for now a correlation not a causal explanation - may have implications for incentives and personal strategies of individual HFP scientists.

\subsection{Interview commentaries on the co-nomination HFP field map}

Interviews with the two HFP scientists helped us not only understand and interpret the conomination data, but also assess the network's validity and the extent to which it represents the intellectual structure of HFP as a research field. Looking at non-anonymised illustrations of the network, both interviewees recognised broad groupings of names of HFP scientists showing ' $a$ clear distinction between experiment and theory' and people in bridging roles 'sitting more between experiment and theory' (I2). They also recognised, named and associated clusters of scientist names with actual sets of HFP experimental equipment around the world. The interviewees also labelled the clusters shown in Table 6. When asked to locate HFP colleagues they knew personally, and to explain why these colleagues might have been co-nominated with specific other scientists, the interviewees provided meaningful narrative interpretations. Each interviewee was also present in the network themselves, and generally agreed their network positions validly represented their own perceived location in the HFP field overall. They could also comprehend and explain to us with whom they were co-nominated and why.

Both interviewees concluded that the co-nomination HFP field map of intellectual influence was valid. They commented it captured people 'definitely in the current flavour physics landscape' (I1), featured 'structures that make sense', and named both 'many well-known experimentalists' (I2) and people widely regarded as 'parents of the field' or 'father' figures,

\footnotetext{
12 The node representing this scientist has the highest eigenvector centrality score in the network (1.0), which means nominations for this person come from other nodes in the network that are also highly nominated.
} 
including one who 'recently retired' (I1). They commented the map also captured known project/collaboration leader roles, and supervisor/supervisee relationships. The interviewees agreed future-looking orientations were also part of the map, such that this co-nomination approach had captured dynamic intellectual trends and potential future directions not just intellectual foundations. When we asked about whether HFP scientists who were most frequently nominated were indeed most intellectually influential, the interviewees agreed this was probably correct as of the time of the interview. Both interviewees also agreed the conomination network was not missing any major intellectual groupings.

Initially both interviewees were surprised certain people they considered had made major contributions to the field appeared not very visible. Upon further inspection these 'prominent figures' were often present in the network, but not central (I1). This appeared to be because these scientists made important contributions in the past and since the frontier of knowledge had advanced. Our interviewees also speculated survey respondents from 'the younger generation' might not have personally interacted with these prominent figures, perhaps explaining their absence from the map (I1). Here we can tentatively conclude names in the conomination network represent the current state and future directions of the HFP research field rather than intellectual history and foundations.

Finally, the interviewees noted a potential underrepresentation of USA and non-Europe HFP scientists. At the same time USA-based authors constituted the largest share of our survey respondents. Our interviewees suggested our co-nomination map may not have 'fully captured heavy flavour physics on an equal footing' (I1) and instead was 'dominated' by one particular 'fashion' of research on possible 'anomalies' and 'anomaly hunting' beyond the Standard Model (I2). Both interviewees agreed the co-nomination map was not comprehensive but nevertheless provided a valid picture of experiment/theory HFP intellectual orientations and connections, captured key intellectual relationships, and reflected social, organisational and intellectual influences (at least within the limits of their own knowledge of HFP as active scientists within the field).

\section{Discussion of the co-nomination approach}

We believe the interviewees' validation of very many aspects of the HFP co-nomination field map suggests the co-nomination approach is a valid contribution to studies of the global science system and its dynamics. This relational, co-nomination approach seemingly can be used as an alternative or complementary tool to map and analyse intellectual influence - especially in hyper-authorship fields like HFP where traditional metrics-based methods display distinct shortcomings. The co-nomination approach highlighted important intellectual structure characteristics of the HFP field, including the theory/experiment divide, the role of connecting and mediating scientists linking theory and experiment, and HEP researchers from proximate fields contributing to HFP.

Added methodological and empirical value of co-nomination becomes apparent when contrasting it with our ACA metrics-based network map of HFP. It is of course acknowledged that bibliometric methods struggle to elicit individual level intellectual influence in hyperauthorship fields (like HFP). However, we also found from our interviews that the ACA map had difficulties to identify and represent correctly intellectual influence and intellectual foundations. Our interviewees suggested the ACA approach captured perhaps overly large numbers of token citations to seminal works and yet perhaps fewer than expected citations to certain field textbooks, manuals and so on, that have become 'so standard that we stop citing them' (I2) (i.e. obliteration by incorporation, c.f. Garfield 1987). The most prominent 
individual figures in the ACA network were scientists who had made significant methodological and computational contributions, whilst scientists who had made monumental past theoretical contributions were less central than perhaps they should have been.

For research fields closely interlinked with other fields and disciplines, like HFP, ACA also does not allow analysis of the knowledge network structure. For HFP it does show HEP is influential over HFP but does not reveal relationships between the various intellectually influential HFP streams. Additionally, authors (or author collectives) who drive ongoing, promising or pioneering research are not sufficiently visible via ACA. This is perhaps because their research has not yet yielded results of certain significance or insufficient time has passed since important discoveries were made for them to start being significantly cited.

Co-nomination seems to provide a more nuanced picture and shows internal intellectual differentiation within the HFP research field. It also identifies more precisely points where HFP links with other proximate research fields. It further bypasses a limitation of bibliometric methods that cannot identify individual-level intellectual influence amongst HFP experimentalists. Our co-nomination approach findings also emphasise the importance of organisational positions within the HFP experiment. These increase the visibility and seeming intellectual influence of individual researchers above and beyond effects related to citation of their published outputs that would be visible via ACA.

We should however note that the co-nomination approach to unpack group authorship and analyse individual-level intellectual influence may be viewed as clashing with HFP field norms. HFP researchers perhaps deliberately de-emphasise or hide individual contributions and together seem to prefer to stress that HEP/HFP research is a collective process (also here justifying costly multi-national investments by member states and their citizens who contribute to this significant collaborative scientific research undertaking). There are potential dangers to using a co-nomination approach in this context. At the same time we feel it remains important to understand mechanisms behind individual reputation-based advancement in fields like HFP. Otherwise perhaps opaque reputation mechanisms in HEP/HFP may disadvantage, for instance, women and/or ethnic minority scientists but yet escape appropriate scrutiny and accountability (Traweek 1988; Birnholtz 2006). ${ }^{13}$ In most research fields we believe important decisions about knowledge and resource arrangements are still made by smaller groups of intellectually influential persons. It is therefore crucial to be able to understand how intellectual influence is accumulated, distributed and mobilised within research fields.

For experimental HFP, we also provide a rare insight into individual-level intellectual influence structure (here closely linked to formal organisational/governance positions in CERN-based experiments). Based on previous knowledge about chain-referral sampled social networks and from our interviews, these may be enduring structural features of the HFP research field, representing structural distribution of intellectual influence. If so, we argue co-nomination analysis as a method is certainly viable to map the intellectual influence network of research fields and has definite utility to analyse such key structural arrangements.

This method of research field mapping using co-nomination, asking members of a field about other intellectually influential scientists, allows us to bypass proxy indicators like citations, and to measure intellectual influences directly. It seems to avoids certain limitations associated with

\footnotetext{
${ }^{13}$ Here we agree with certain scholars arguing against Knorr-Cetina's metaphor that HEP has become a simple 'production line' (Knorr-Cetina 1999).
} 
citation-based analyses, such as differences in citing behaviour, or that ACA in particular usually selects a sample of the most cited authors, which is an unhelpful bias when there are multiple 'schools of thought' or competing intellectual traditions in a research field (Nicolaisen 2006) - such as HFP theory/experiment divides, and differing HFP experiment structures.

\section{Conclusions}

Using co-nomination to map research fields appears analytically and intellectually useful. It seemingly can substitute or complement bibliometric-based techniques like ACA, in specific ways and instances, as we have demonstrated here for the particular hyper-authorship, massively collaborative case of the HFP research field.

We can also discuss potential uses of this approach for science policy studies and research evaluation arrangements in the global science system. First, caution should be exercised around analysis of specific people's names generated in the co-nomination network. Some scientists may appear influential at a certain instance by researching currently trendy or promising topics, but such influence may wax and wane along with fads and fashions common to the frontier dynamics of most research fields. Stressing particular scientist names and the frequency with which individual scientists are nominated cannot, and we believe should not, be used for any form of evaluation of short- or long-term contribution to a field.

Certain shortcomings of our co-nomination approach are mainly found in the starter set sampling and response bias that are common to most survey-based methodologies. Our results nevertheless seem representative of the HFP field knowledge network's intellectual influence structure. At the same time peripheral nodes may not be as peripheral as they perhaps appear. Co-nomination certainly seems prone to reflect intellectual influence of current researchers in the field rather than researchers who have made significant past contributions but are no longer active. Traditional citation-based methods like ACA may be more suited here to explore this aspect of intellectual influence. Co-nomination is also far more resource- and time-intensive to use, and is not an unobtrusive technique in contrast to approaches like ACA.

Key advantages of co-nomination are that it can map knowledge networks in fields where academics do not only publish in peer-reviewed journals (i.e. HEP/HFP pre-print culture), and those not adhering to typical author credit conventions (i.e. HEP/HFP hyper-authorship with mandated alphabetical ordering). For the future, co-nomination might also be used to map other research fields with hyper-authorship, such as space science, molecular biology and genetics or biomedicine (Adams et al. 2019; Cronin 2001). It could also be used for research fields where books are the primary research outputs, for fields where intellectual influence centres around grey literature/reports, and perhaps for fields with significant shares of literature published in non-English languages (i.e. given metrics-based methods primarily draw upon English language publication databases). Lastly, co-nomination might also be considered for mapping intellectual influence around issues beyond purely research, e.g. contemporary university-industry-government policy and scientific processes involving input from not only scientists but also lay experts and other stakeholders. 


\section{References}

Adams, Jonathan, David Pendlebury, Ross Potter, and Martin Szomszor. 2019. Global research report: Multi-authorship and research analytics. Beijing, China, London, UK, Tokyo, Japan, Philadelphia, USA: Clarivate Analytics Institute for Scientific Information Web of Science Group, ISBN 978-1-9160868-6-9.

Beauty Workshop 2014. CERN Indico. https://indico.cern.ch/event/308116/. Accessed 19 Dec 2019.

Beauty Workshop 2016. CERN Indico. https://indico.cern.ch/event/352928/. Accessed 19 Dec 2019.

Bellotti, E. 2011. The social processes of production and validation of knowledge in particle physics: Preliminary theoretical and methodological observations, in: Cronin, B., and D. Christopoulos (Eds.), 4th and 5th UK Social Networks Conferences. Amsterdam, Netherlands: Elsevier Science Bv.

Birnholtz, Jeremy P. 2008. When authorship isn't enough: Lessons from CERN on the implications of formal and informal credit attribution mechanisms in collaborative research. The Journal of Electronic Publishing: 11(1), 1-8, http://dx.doi.org/10.3998/3336451.0011.105m

Birnholtz, Jeremy P. 2006. What does it mean to be an author? The intersection of credit, contribution, and collaboration in science. Journal of the American Society for Information Science and Technology 57: 1758-1770. https://doi.org/10.1002/asi.20380.

Blondel, Vincent D., Jean-Loup Guillaume, Renaud Lambiotte, and Etienne Lefebvre. 2008. Fast unfolding of communities in large networks. Journal of Statistical Mechanics: Theory and Experiment 2008: P10008. https://doi.org/10.1088/1742-5468/2008/10/P10008.

Borgatti, S. P., M. G. Everett, and J. Johnson. 2018. Analyzing Social Networks. Los Angeles and Washington, DC, USA, London, UK, New Delhi, India, Singapore: SAGE Publications.

Boyack, K.W., and R. Klavans. 2014. Creation of a highly detailed, dynamic, global model and map of science. Journal of the Association for Information Science and Technology 65: 670-685. Scopus. https://doi.org/10.1002/asi.22990.

Braam, R., and P. van den Besselaar. 2014. Indicators for the dynamics of research organizations: a biomedical case study. Scientometrics 99: 949-971.

https://doi.org/10.1007/s11192-014-1235-5

Braun, Dietmar. 2012. Why do scientists migrate? A diffusion model. Minerva 50: 471-491. https://doi.org/10.1007/s11024-012-9214-6.

Canals, A., E. Ortoll, and M. Nordberg. 2017. Collaboration Networks in Big Science: The ATLAS Experiment at CERN. Profesional De La Informacion 26: 961-971. https://doi.org/10.3145/epi.2017.sep.17

Campanelli, Mario. 2015. Inside Cern's Large Hadron Collider: From The Proton To The Higgs Boson. New Jersey, USA: Wspc.

CHARM Workshop 2013. http://www.charm2013.manchester.ac.uk/. Accessed 19 Dec 2019. 
CHARM Workshop 2015. https://charm2015.wayne.edu. Accessed 19 Dec 2019.

CHARM Workshop 2016. http://charm2016.bo.infn.it/index.php. Accessed 19 Dec 2019.

CKM Workshop 2014. http://ckm2014.hephy.at/home/. Accessed 19 Dec 19.

CKM Workshop 2016. https://www.tifr.res.in/ ckm16/. Accessed 19 Dec 19.

Crane, Diana. 1971. Transnational Networks in Basic Science. International Organization 25: 585-601. https://doi.org/10.1017/S0020818300026333.

Creswell, John W. 2009. Editorial: Mapping the Field of Mixed Methods Research. Journal of Mixed Methods Research 3: 95-108. https://doi.org/10.1177/1558689808330883.

Cronin, Blaise. 2001. Hyperauthorship: A postmodern perversion or evidence of a structural shift in scholarly communication practices? Journal of the American Society for Information Science and Technology 52: 558-569. https://doi.org/10.1002/asi.1097.

Degn, L., N. Mejlgaard, N., and J.W. Schneider. 2019. Using mixed methods to map vaguely defined research areas. Research Evaluation. 28(4): 394-404.

https://doi.org/10.1093/reseval/rvz025 .

Fagerberg, Jan, and Bart Verspagen. 2009. Innovation studies-The emerging structure of a new scientific field. Research Policy 38: 218-233.

https://doi.org/10.1016/j.respol.2008.12.006.

Georghiou, L. 1998. Global cooperation in research. Research Policy 27: 611-626.

https://doi.org/10.1016/S0048-7333(98)00054-7.

Gershon, T., and M. Needham. 2015. Heavy Flavour Physics at the LHC. Comptes Rendus Physique 16: 435-447. https://doi.org/10.1016/j.crhy.2015.04.001.

Haas, Peter M. 1992. Introduction: epistemic communities and international policy coordination. International Organization 46: 1-35.

https://doi.org/10.1017/S0020818300001442.

Heimeriks, Gaston, Marianne Hörlesberger, and Peter Van Den Besselaar. 2003. Mapping communication and collaboration in heterogeneous research networks. Scientometrics 58: 391-413. https://doi.org/10.1023/A:1026296812830.

Heuer, Rolf-Dieter. 2012. The future of the Large Hadron Collider and CERN. Philosophical Transactions of the Royal Society a-Mathematical Physical and Engineering Sciences 370: 986-994. https://doi.org/10.1098/rsta.2011.0467.

Johnson, Stephen C. 1967. Hierarchical clustering schemes. Psychometrika 32: 241-254. https://doi.org/10.1007/BF02289588.

Knorr-Cetina, Karin D. 1999. Epistemic Cultures: How the Sciences Make Knowledge. Boston, MA, USA: Harvard University Press.

Knorr-Cetina, Karin D. 1982. Scientific Communities or Transepistemic Arenas of Research? A Critique of Quasi-Economic Models of Science. Social Studies of Science 12: 101-130. https://doi.org/10.1177/030631282012001005. 
Lambert, Robert W. 2011. Heavy Flavour in a Nutshell. arXiv preprint arXiv:1105.1061.

Langfeldt, Liv, Maria Nedeva, Sverker Sörlin, and Duncan A. Thomas. 2020. Co-existing Notions of Research Quality: A Framework to Study Context-specific Understandings of Good Research. Minerva 58, 115-137. https://doi.org/10.1007/s11024-019-09385-2.

Lee, Woo. 2008. How to identify emerging research fields using scientometrics: An example in the field of Information Security. Scientometrics 76: 503-525.

https://doi.org/10.1007/s11192-007-1898-2.

Lehmann, S., B. Lautrup, and A. D. Jackson. 2003. Citation networks in high energy physics. Physical Review E 68: 026113. https://doi.org/10.1103/PhysRevE.68.026113.

Libbey, Miles A., and Gerald Zaltman. 1967. The role and distribution of written informal communication in theoretical high energy physics. Available from A1P as Report No.

AIP/SDD-1(REV.), also USAEC Report No. NYO-3732-1(REV.).

Luukkonen, Terttu, and Maria Nedeva. 2010. Towards understanding integration in research and research policy. Research Policy 39(5): 674-686.

Meho, Lokman I., and Kiduk Yang. 2007. Impact of data sources on citation counts and rankings of LIS faculty: Web of Science versus Scopus and Google Scholar. Journal of the American Society for Information Science and Technology 58: 2105-2125. https://doi.org/10.1002/asi.20677.

Merton, R.K. 1968. Social Theory and Social Structure. New York, USA: Simon and Schuster.

Nedeva, Maria. 2013. Between the global and the national: Organising European science. Research Policy 42: 220-230. https://doi.org/10.1016/j.respol.2012.07.006.

Nedeva Maria. 2010. Public Sciences and Change: Science Dynamics Revisited, in Janusz Mucha and Katarzyna Leszczynska (eds) Society, Culture and Technology at the Dawn of the $21^{\text {st }}$ Century, Cambridge Scholars Publishing: Cambridge; ISBN 978-1-4438-2156-8

Nedeva, Maria, Luke Georghiou, Denis Loveridge, and Hugh Cameron. 1996. The use of conomination to identify expert participants for Technology Foresight. R\&D Management 26: 155-168. https://doi.org/10.1111/j.1467-9310.1996.tb00939.x.

Nerur, Sridhar P., Abdul A. Rasheed, and Vivek Natarajan. 2008. The intellectual structure of the strategic management field: An author co-citation analysis. Strategic Management Journal 29: 319-336. https://doi.org/10.1002/smj.659.

Nicolaisen, Jeppe. 2006. Traditional author co-citation analysis: A discussion of the sampling problem. In: International Conference on Multidisciplinary Information Sciences and Technologies. Merida, Spain.

Noyons, C.M. 2004. Science Maps within a Science Policy Context, in H. Moed, W. Glänzel, and U. Schmoch (Eds.): Handbook of Quantitative Science and Technology Research. Dordrecht, Germany: Springer, 237-255. https://doi.org/10.1007/1-4020-2755-9_11 
Porter, Alan, and Ismael Rafols. 2009. Is science becoming more interdisciplinary? Measuring and mapping six research fields over time. Scientometrics 81: 719-745. https://doi.org/10.1007/s11192-008-2197-2.

Saritas, O., and Y. Nugroho. 2012. Mapping issues and envisaging futures: An evolutionary scenario approach. Technological Forecasting and Social Change. 79(3): 509-529. https://doi.org/10.1016/j.techfore.2011.09.005.

Shrum, W., and N. Mullins. 1988. Network Analysis in the Study of Science and Technology. In A. F. J. van Raan (Ed.): Handbook of Quantitative Studies of Science and Technology, Amsterdam, Netherlands, New York, USA, Oxford, UK, Tokyo, Japan: Elsevier, North Holland, 107-134.

Storer, N. W. 1966. The Social System of Science. New York, USA: Holt, Rinehart and Winston.

Traweek, Sharon. 1988. Beamtimes and Lifetimes. Boston, MA, USA: Harvard University Press.

Van Raan, A. F. J., and R. J. W. Tijssen. 1993. The neural net of neural network research. Scientometrics 26: 169-192. https://doi.org/10.1007/BF02016799.

Wejnert, Cyprian. 2010. Social network analysis with respondent-driven sampling data: A study of racial integration on campus. Social Networks 32(2): 112-124.

https://doi.org/10.1016/j.socnet.2009.09.002.

Wellman, B., and Berkowitz, S.D..1988. Introduction: Studying social structures, in B.

Wellman, S.D. Berkowitz, and M. Granovetter (Eds.) Social structures: A network approach. Cambridge, UK: Cambridge University Press, 1-14.

White, H. D., and K. W. McCain. 1998. Visualizing a discipline: An author co-citation analysis of information science, 1972-1995. Journal of the American Society for Information Science 49: 327-355. https://doi.org/10.1002/(SICI)1097-4571(19980401)49:4<327::AIDASI4>3.0.CO;2-W.

White, Howard D., and Belver C. Griffith. 1981. Author cocitation: A literature measure of intellectual structure. Journal of the American Society for Information Science 32: 163-171. https://doi.org/10.1002/asi.4630320302.

Whitley, Richard. 2000. The Intellectual and Social Organization of the Sciences. Oxford, UK: Oxford University Press.

Zhao, Dangzhi, and Andreas Strotmann. 2008. Author bibliographic coupling: Another approach to citation-based author knowledge network analysis. Proceedings of the American Society for Information Science and Technology 45: 1-10. https://doi.org/10.1002/meet.2008.1450450292. 


\title{
Appendix 1. Author Co-Citation Search Query for Heavy Flavour Physics Articles in the Web of Science Database
}

\author{
TS=(((charm* OR bottom* OR beauty*) AND (quark OR meson* OR mixing OR "rare \\ decays")) OR ("b quark*" OR "c quark*" OR "b meson*" OR "c meson*") OR ("b-quark*" \\ OR "c-quark*") OR ("heavy flavour" OR "heavy flavor")) AND WC=(Physics, Particles \& \\ Fields) AND LANGUAGE: (English) AND DOCUMENT TYPES: (Article)
}

